Methodik der Nachhaltigen Entwicklung

\title{
Systemische Strukturaufstellungen als neue Methode zur Förderung einer nachhaltigen Entwicklung
}

\author{
Um eine nachhaltige Entwicklung zu fördern \\ und adäquat umzusetzen, bedarf es neuer \\ Methoden und Instrumente. In inter- und \\ transdisziplinären Zusammenhängen können \\ mithilfe von Systemischen Strukturaufstellungen \\ in Science-private-Partnerships, neue und \\ nachhaltige Impulse und Lösungsstrategien \\ entdeckt und entwickelt werden. \\ Von Marlen Arnold
}

\section{Einführung}

Die Realisierung einer nachhaltigen Entwicklung steckt trotz unseres umfangreichen Wissens noch immer in den Kinderschuhen. Zugleich scheinen die herkömmlichen wissenschaftlichen Ansätze und Praktiken begrenzt zu sein, da sie oft nicht gewiss und umfassend die Wirklichkeit, aktuelles Verhalten, strategische Entscheidungen oder die Resilienz eines Systems erklären können (Walker et al. 2002). Die heutige Unternehmensführung ist stärker denn je in multikausalen sowie inter- und transdisziplinären Zusammenhängen aktiv, die auch neue und innovative Methoden erfordern, um eine nachhaltige Entwicklung zu fördern. In Systemischen Strukturaufstellungen (SyStA) können Systeme durch räumliche Anordnungen von Personen oder Symbolen abgebildet und simuliert werden. Die Methode ist der Aktionsforschung (Schlötter 2005) zuzurechnen. Ihr Kern besteht aus der repräsentierenden Wahrnehmung und dem systematischen räumlichen Positionieren von Entscheidungsträgern im Raum (Varga von Kibéd et al. 2014; Müller-Christ 2012). SyStA erlauben sowohl einen tieferen Blick in die informellen Strukturen und Beziehungen und sozialen Gefüge von Institutionen als auch das Prüfen oder Testen von Maßnahmen und verschiedenen Lösungsmöglichkeiten im Hinblick auf ihre (Aus-)Wirkungen (Sparrer et al. 2001). Dieser Artikel gibt einen Einblick in die transdisziplinäre Wirksamkeit des Verfahrens und zeigt wichtige Ergebnisse und Einsatzmöglichkeiten für transdisziplinäre Lehre und Forschung sowie die Anwendung in nachhaltigkeitsbezogenen Beratungskontexten. Im Artikel wird ein konkretes Beispiel aus sogenannten Science-private-Partnerships vorgestellt, bei denen SyStA im Rahmen von Kooperationen zwischen Forschungseinheiten und Praxisvertreter/innen zur Anwendung kommen. Der Nutzen für die drei Bereiche Forschung, Lehre und Praxis wird kritisch diskutiert.

\section{Systemische Strukturaufstellungen - Was ist das?}

Laut Wade (2004) bieten Strukturaufstellungen leistungsstarke und kreative Wege zur Klärung und Lösung von komplexen, möglicherweise unlösbaren Problemen in organisationalen Kontexten, Systemen oder mit gesellschaftlichen Akteuren. SyStA vermögen Muster, Zusammenhänge, Strukturen, Beziehungen innerhalb eines Systems räumlich abzubilden und können so vielfältig eingesetzt werden (Kopp 2013). SyStA bieten einen strukturierten Weg, eine Masse von Informationen und Daten, Details und Meinungen zu fokussieren sowie Aspekte in einer neuen Dimension zu pointieren. Wade (2004) sieht das Potenzial sowohl in der Klarheit als auch im Experimentieren mit möglichen Optionen in einer sicheren Umgebung, um so die Entscheidungsfindung zu unterstützen. Die Aufstellungsarbeit hat vielfältige Wurzeln (Daimler 2014), wie etwa die Familiensystemtherapie, Lösungsfokussierung oder existenzielle Phänomenologie-Ansätze. Heute gibt es eine breite Anwendung dieser Methode in verschiedenen Kontexten wie Psychologie, Medizin, Pädagogik, Ökonomie und so weiter. Strukturaufstellungen basieren auch auf der Systemtheorie und heben so die Idee hervor, dass Systeme in erster Linie sich selbst regulierende Entitäten sind und immer nach Ausgleich suchen sowie in der Lage sind, sich zu ändern oder zu transformieren. Bestimmte Elemente gelten als systemrelevant. Der Zugewinn systemischer Betrachtungsweisen liegt vor allem darin, die Überlagerung mehrerer logischer Ketten und Beschreibungsebenen aufzuzeigen.

In der Praxis gibt es eine Vielzahl von Aufstellungsformaten (Varga von Kibéd et al. 2014; Daimler 2014). Dennoch ist das Verfahren nicht beliebig, sondern hat bestimmte Regeln und Strukturen. Im Allgemeinen gibt es drei verschiedene Funktionen in Strukturaufstellungen: Aufstellungsleitende beziehungsweise Prozessbegleitende, Anliegengebende und eine Gruppe von Menschen, die bereit ist, als Stellvertretende mitzuwirken. Ein Aufstellungsprozess beginnt mit der Klärung des Anliegens zwischen Aufsteller/in und Anliegengeber/in: 
Was für ein Ziel soll erreicht und welche Schlüsselelemente des spezifischen Systems sollen einbezogen werden? Diese wählt der/die Anliegengeber/in nach der eigenen Intuition aus dem Kreis der vorhandenen Personen aus. Schließlich werden alle Repräsentant/innen nach Gefühl oder innerem Bild im Raum von der/dem Anliegengebende/n gestellt. Einmal platziert, können die Repräsentant/innen wichtige Informationen bezüglich des aufgestellten Systems liefern (Franke 2003). Zur Prozessgestaltung bittet die/der Aufsteller/in die Repräsentant/ innen entweder ihren Bewegungsimpulsen zu folgen und/oder fragt die Stellvertretenden nach Befinden und Unterschieden. Der Einsatz von gewaltfreier Kommunikation (Rosenberg 2013) sollte fester Bestandteil der Prozessführung sein. Wesentlich für das Verfahren ist die Beschreibung von Körperwahrnehmungen, Emotionen, aufkommenden Bildern oder Sätzen ohne Interpretation und Bewertung beziehungsweise vorherige Selektion durch die Repräsentant/innen. Nachdem ein erstes Systembild vorhanden ist, können sich die laufenden Prozesse und Interventionen je Schule, Aufstellungsformat und Aufstellungsleitung unterscheiden.

Der Aufstellungsprozess ist in der Regel abgeschlossen, wenn ein Lösungsbild gefunden ist, mit dem der/die Auftraggebende zufrieden ist, oder der Prozess keine Weiterentwicklung erzielt. Aufstellungen dauern zwischen 20 Minuten und zwei Stunden. Die Repräsentant/innen werden aus ihrer Funktion entlassen und der/die Auftraggeber/in dankt ihnen. Abschließend gibt es eine Nachbesprechung zwischen Aufsteller/in und Auftraggeber/in. Eine kompetente und verantwortungsvolle Aufstellungsleitung basiert auf einer mehrjährigen Ausbildung und Zertifizierung, die so auch die Qualität sichern soll.

\section{Systemische Strukturaufstellungen im Kontext von Organisationen}

In organisationalen Kontexten sind verschiedene Ebenen zu unterscheiden, die einen Einfluss auf die aktuelle Situation und die Lösung haben. Da Menschen stets Teil der Haupttransaktions- und Austauschprozesse sind, ist auch eine persönliche Ebene wirksam. Aufstellende sollten jedoch immer auch die Organisationsebene sowie die noch komplexere Systemebene im Blick haben, denn es gibt vielfältige moderierende Faktoren und Zusammenhänge.

\subsection{Potenzielle Fragestellungen}

Eine Auswahl an potenziellen Fragestellungen der drei Ebenen sind folgend aufgeführt:

\section{Ebene der Individuen}

- Wie wird die Situation durch persönliche Erfahrungen und Muster der Mitarbeitenden, die ihren Ursprung in ihrer Geschichte, der Lebenserfahrungen oder Dynamik in den Familien haben, beeinflusst? Finden diese Muster möglicherweise innerhalb der Organisation wieder Wirkung?
- Haben Beziehungskonflikte oder problematische Verhaltens- und Kommunikationsmuster der Mitarbeitenden oder zwischen Abteilungen negative Auswirkungen auf die Organisation?

\section{Organisationsebene}

- Hat die Organisation funktionale Strukturen? Oder signalisieren auftretende Schwierigkeiten auf der Beziehungsebene grundlegende Probleme des organisationalen Systems?

- Haben alle Menschen ihren angemessenen Platz?

- Sind Führungs- und Managementaufgaben angemessen implementiert und ausgeübt?

- Welche Rolle nehmen Managementsysteme in der Institution ein und wie lassen sie sich ändern?

- Wie steht es um Innovation und Kultur?

\section{Systemebene}

- Gab oder gibt es Veränderungen in der Umwelt, sodass neue Herausforderungen anstehen?

- Wie sind die Historie und die Gründung des Unternehmens?

- Wie werden kulturelle Unterschiede gemanagt und integriert?

- Sind die bestehenden Managementsysteme für die gesamte Wertschöpfungskette angemessen?

- Wie ist der Umgang mit Transformation?

Systemdynamiken folgen gewissen Ordnungen und Prinzipien (Daimler 2014; Varga von Kibéd et al. 2014; Sparrer et al. 2010; Sparrer et al. 2001). Dabei gibt es sowohl Gemeinsamkeiten als auch Unterschiede zwischen Familien- und Organisationssystemen. Ein direkter Transfer ist begrenzt.

\subsection{Aspekte der Systemdynamiken}

Nach König (2007) sollten die folgenden Aspekte berücksichtigt werden:

Zugehörigkeit und Ausschlusskriterien. Eine Organisationszugehörigkeit ist vorübergehend erworben und kann von beiden Seiten gekündigt werden. Nur dies bewirkt die transpersonale Kontinuität der Organisationen.

Vorrang im System und im Vergleich zu anderen Systemen. In Familiensystemen gibt es vertikale und horizontale Strukturen, hierarchische und zeitliche Logiken. Horizontale Ordnungen können in Familiensystemen nicht verändert werden, wohingegen die vertikalen Strukturen durch die Gründung einer Familie geändert werden. In Organisationen sind vertikale Strukturen und Positionierungen in Bezug auf das Dienstalter vorübergehend und nur auf die professionelle Rolle einer Person ausgerichtet. Positionen sind zugewiesen und nicht natürlich gegeben.

Hierarchie der Positionen und Rollen in einer Organisation. Hierarchien werden durch verschiedene Aspekte in Unternehmen beeinflusst. Führung, Zuständigkeiten, Aufgaben, Kompetenz, Erfahrung und Zeit der Mitgliedschaft in einer Organisation sind von hoher Bedeutung in Managementkontexten. 
Mehrdeutigkeit kostet immer Energie. Versteckte Hierarchien sind zu vermeiden. Zudem gibt es weitere Mikro-, Meso- und Makroebeneneffekte, welche die organisatorische Realität beeinflussen.

Modalitäten des Gebens und Nehmens und damit verbundene Machtbeziehungen, Beziehungen und Verpflichtungen. Altruismus und Liebe sind Teil der Hauptsystemlogik von Familien. Die zentralen Logiken in organisatorischen Kontexten sind Gewinne und Löhne (Geld). Der Saldo aus Geben und Nehmen ist mehr im Zusammenhang mit der Familie bedeutsam, während die Hauptkompensation in Organisationen auf dem Tauschhandel basiert. Diese Art von Austausch oder Gerechtigkeit kann in Familiensystemen über mehrere Generationen gelten. Eine Idee, die sich im Wirtschaftsleben auch über die Internalisierung der externen Effekte verankern lässt.

Engagement, Leistung und deren Anerkennung - je größer das Engagement, desto mehr zu sagen. Organisationen müssen Veränderung dauerhaft begegnen, demzufolge sollten sie besonderes Engagement ihrer Mitglieder honorieren. Leistung sollte dort gewürdigt zugewiesen werden, wo sie entsteht. Auch die langfristige Leistungsstärke der Mitglieder ist zu würdigen. Allerdings haben höhere Leistungen und Fähigkeiten Priorität.

Funktionalität innen und außen - Letzteres hat eine dominantere Stellung. In ökonomischen Kontexten kann es sinnvoller sein, die Aufstellungsarbeit dazu zu nutzen, das Verständnis und die Funktionalität der gegebenen Führung und so weiter auf Grundlage des Feedbacks der Organisationsmitglieder und vorhandener Informationen zu erhöhen und zu prüfen, anstatt an Vorstellungen von Ordnungen festzuhalten. König (2007) hebt hervor, dass der Unterschied zwischen innen und außen nur eine relevante Unterscheidung von mehreren ist. Es sollten weitere Unterscheidungen gemacht werden, um die Situation und die Konsequenzen sichtbar und spürbar herauszuarbeiten, zur Lösungsfindung und um den Raum von Optionen zu verbessern.

Vergänglichkeit - wenn ein Organisationssystem seine Aufgabe verliert, muss es auch die Möglichkeit geben, es aufzulösen. Es gibt einen grundsätzlichen Unterschied in Bezug auf Zeit. Die zeitliche Reihenfolge innerhalb der Familie wird direkt mit unserer Lebenszeit verbunden; während Organisationen diese Einschränkung nicht haben. Beide Systeme haben verschiedene Zeithorizonte.

\section{Methodische Reflexionen zu Systemischen Strukturaufstellungen}

Mithilfe der Methode lassen sich implizites Wissen sowie versteckte oder zugrunde liegende, jedoch nicht bewusst wahrgenommene Dynamiken aufzeigen. Charakteristisch für die repräsentierende Wahrnehmung (Sparrer et al. 2010) ist, dass die in den Raum gestellten Personen (Stellvertretende, Repräsentant/innen) Körpersymptome und Gefühle wahrnehmen, die nicht zu ihnen selbst, sondern zur Person beziehungsweise dem System der Person gehören, für die sie stellvertretend ste- hen. Menschen, welche die Methode der SyStA nicht kennen, reagieren häufig befremdlich, mit Unverständnis und Zweifel auf die Beschreibung der repräsentierenden Wahrnehmung. Diese anfängliche Skepsis lässt sich häufig reduzieren, indem die Stellvertretung und die damit verbundenen Fremdwahrnehmungen und -gefühle selbst erlebt werden.

Wie genau die repräsentierende Wahrnehmung funktioniert, wird noch aktiv beforscht und ist noch nicht verstanden. Eine Grundannahme basiert darauf, dass die repräsentierende Wahrnehmung der jeweiligen Stellvertretenden nicht zufällig oder beliebig ist oder gar einem Skript folgt, sondern durch die Position in der Aufstellung selbst bestimmt ist. Schlötter (2005) hat dazu in einem empirischen Versuchsdesign, das den gängigen wissenschaftlichen Qualitätskriterien entspricht, nachgewiesen, dass die Wahrnehmung der Position im Raum eine Art nicht verbale Sprache der Stellung von Personen im Raum zueinander darstellt. Es handelt sich um eine Art Zeichensprache oder Zeichensystem, vergleichbar einer Sprache, die einer allgemein verständlichen Semantik folgt. Hervorzuheben ist, dass die repräsentierende Wahrnehmung unabhängig von Person, Sprache und Kultur funktioniert. Der englische Biologe Ruppert Sheldrake $(1988 ; 2001)$ prägte den Begriff des „Morphischen Feldes“ beziehungsweise der "Morphischen Resonanz", befähigt, Informationen festzuhalten. Diese Felder sind weitgehend unabhängig von Raum und Zeit und ermöglichen eine Art Erinnerung.

Ein weiterer Erklärungsversuch bezieht sich auf die Quantenfeldtheorie. Gehlert (2014) betont, dass Greenberg et al. (1990; 2008) die Verschränkung und Quanteninformationsübertragung in Mehrpartikelsystemen beschreiben. In den Experimenten werden beliebig vielteilige Quantenfelder ermittelt. Gehlert (2014) gründet seine weitere Argumentation nun auf der Annahme, dass lebende Organismen aufgrund der metastabilen, elektromagnetischen Struktur ihrer Grundelemente (Atome, Moleküle, Synapsen) sowie ihrer Interaktion makroskopische Quantenfelder darstellen. Dann kann die Art der Systemaufstellungsarbeit analog in Beziehung gesetzt werden und auf der Grundlage der Naturwissenschaften verständlich erklärt werden. Das bedeutet, dass Mehrpartikelsysteme auch Quantenfelder sind und Informationen verbreitet und sofort verfügbar sind. Im Quantenfeld ist alles miteinander verbunden und alles kann mit allem kommunizieren. Der Autor argumentiert auch, dass nur der/die Beobachter/in alle Informationen selbst interpretiert und sämtliche Interpretationen kontextbezogen sind. Mit SyStA ist folglich auch eine Metakommunikation über soziale Systeme möglich und bietet eine Form der Selbstreflexion über soziale Systeme zur Problemlösung.

SyStA sind Teil der qualitativen Forschung, die explorativ und hypothesengenerierend ist. Sie lassen sich der Feld- und Aktionsforschung zuordnen (Mayring 2002; Spiess 1994). Sie eignen sich für organisationale Kontexte, bei denen wenige Informationen vorliegen oder gängige Theorien und Konzepte an ihre Grenzen gestoßen sind. Durch die Raumsprache und das 
Aufzeigen verdeckter Informationen in 3-D-Bildern, kann Abduktion zum Tragen kommen. Gemäß Reichertz (1999, S. 54) ist Abduktion ein mentaler Prozess, „ein geistiger Akt, ein gedanklicher Sprung, der das zusammenbringt, von dem man nie dachte, dass es zusammengehört“. Er stellt klar, dass erst das Ergebnis der Abduktion eine sprachliche Hypothese ist und aus Prozessen resultiert, die weder rational begründbar noch kritisierbar sind. Diese Prozesse werden durch SyStA begünstigt, da oftmals neue Konstellationen sichtbar und damit neue Betrachtungsweisen möglich werden. Systemaufstellungen sollten regelgeleitet und ohne Lösungsinteresse der Aufstellungsleitung ablaufen. In diesem Sinne sind sie ein zuverlässiges Verfahren.

Objektivität, Reliabilität und Validität gelten als Qualitätskriterien wissenschaftlichen Arbeitens (Mayring 2002). Gemäß Schlötter (2005) weisen SyStA eine hohe Objektivität auf. Die Anwendung erfolgt regelgeleitet und ist über Aufstellungsformate detailliert und eindeutig dargestellt (Varga von Kibéd et al. 2014). Forschung zur Reliabilität von Aufstellungen ist sehr aufwendig (Schlötter 2005). Schlötter (2005) konnte in seinen Paralleltests mit unterschiedlichen Proband/innen eine hohe Reliabilität abbilden. In reliabeln Settings erzeugen Systemaufstellungen verlässliche Informationen über implizites oder unbewusstes Wissen, um diese zu expliziten Erkenntnissen zu transformieren. Eine höhere Varianz kann sich ergeben, da die Ausgangssituation nie wieder herstellbar ist. Die interne Validität ist eng an die Reliabilität gebunden (Lamnek 1988). Hier kommt auch das bekannte „Reliabilität-Validität-Dilemma“ zum Tragen. Bei der externen Validität ist stets zu berücksichti- gen, dass in Systemaufstellungen auch die Anliegengebenden die Wirksamkeit einschätzen: Waren die Aussagen hilfreich, um sozial-ökonomische Prozesse gesünder, nachhaltiger beziehungsweise effektiver zu gestalten?

Damit sich implizites Wissen in SyStA tragfähig zeigen kann, braucht die Methode die Verlässlichkeit der Gütekriterien. Die Balance aller Gütekriterien ist entscheidend. Eine hohe Validität ist stets über die Bestätigung des Systembildes durch die Anliegengeber/innen zu sichern. Anliegenfreie Aufstellungen bedürfen einer Achtsamkeit der Interpretation, da unklar ist, was sich zeigt. Die Systemaufstellungen können einen Weg hin zu einem neuen Wissenschaftsverständnis ebnen und die Abduktion wieder stärker ins Blickfeld rücken.

\section{Beispiele aus der Praxis}

Die Wirkkraft von SyStA werden insbesondere dann deutlich, wenn sie das Potenzial impliziten Wissens über konkrete Beispiele veranschaulicht. Nachfolgend soll anhand der Wasserwirtschaft aufgezeigt werden, wie über verschiedene Formate von SyStA neue Ideen, Veränderungsimpulse und nachhaltigere Strategien entstehen können. Konkret stand die Frage im Raum, wie ein Netzwerkengagement zur Stärkung nachhaltigen Wirtschaftens gefördert werden kann und wie sich die Unternehmung im Branchengefüge positioniert. Ziel war auch, herauszuarbeiten, wie, durch wen und mit welcher Botschaft das Netzwerk gegründet werden und auf welche Zielgruppe sich das Netzwerk ausrichten soll, um eine nachhaltige Entwicklung in der Wasserwirtschaft zu stärken. Die Aufstellung

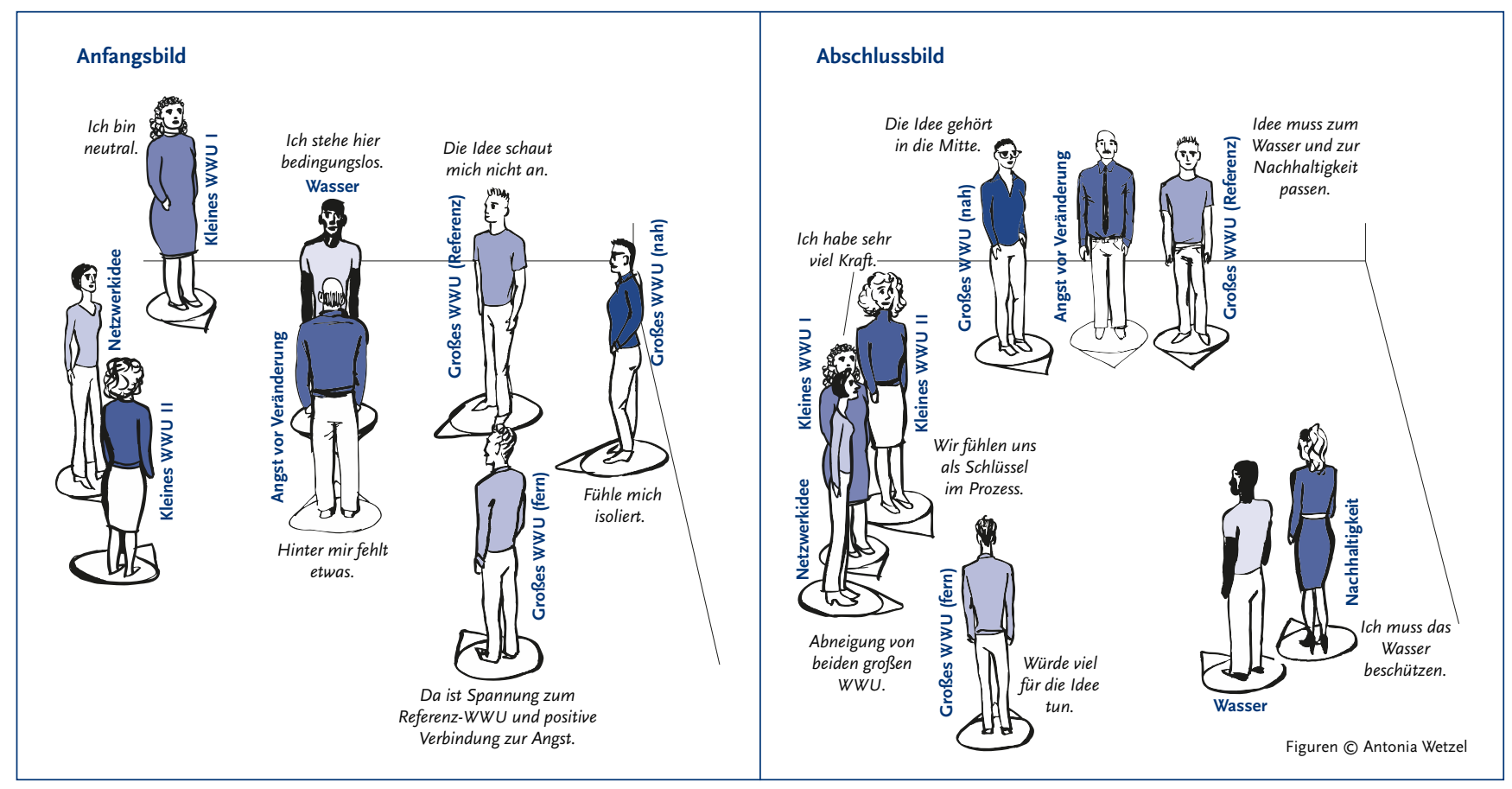

Abbildung 1: Eindrücke einer SyStA im Themenfeld der Wasserwirtschaft 
wurde im Herbst 2014 mit einem Unternehmensvertreter in Leitungsposition durchgeführt. Als Stellvertreter/innen standen Studierende und Mitarbeitende der Universität Bremen zur Verfügung. Startidee war die Stärkung von Nachhaltigkeit in der Wasserwirtschaft. Mittels der Aufstellung konnte erarbeitet werden, dass das Netzwerk mit der Idee starten sollte, reines Wasser bereitzustellen, und weitere Ziele mit Blick auf eine nachhaltige Entwicklung gemeinsam im Netzwerk erarbeiten sollte. Nachhaltigkeit wird als ein zu vages Konzept wahrgenommen, das im Kontext der Wasserversorgung einer verstärkten Klarheit und der Verhandlung spezifischer Strategieoptionen bedarf.

Die Aufstellung wurde wie folgt organisiert: In einem kurzen Auftragsklärungsgespräch wurden das Anliegen, die Systemgrenzen sowie die zu stellenden Elemente geklärt. Der Unternehmensvertreter eines Wasserwirtschaftsunternehmens hat dann die Stellvertreter/innen ausgewählt und in den Raum geführt. Alle Stellvertreter/innen erhielten die Möglichkeit, ihre Wahrnehmungen und Bewegungsimpulse auszudrücken und diesen nachzugehen. Wasser konnte sich als ein freies Element so bewegen, wie die inneren Impulse kamen. Der Austausch und die Bewegung der Repräsentant/innen untereinander zeigte, dass eine gemeinsame Mission fehlt, sodass es keine gute Idee zu sein schien, ein neues nachhaltigkeitsausgerichtetes Netzwerk mit ausgewählten Partnern zu gründen. Innerhalb des Prozesses wurde auch die Angst vor Veränderungen deutlich, die sowohl extern (Fracking) bedingt sein kann als auch auf internen Prozessen beruht (siehe Abb.1). Die Repräsentant/innen signalisierten, dass ein Netzwerk eine klare positive Veränderung für die jeweiligen Unternehmen ermöglichen muss und keinesfalls bisherige Standards aufweichen darf. Während des Prozesses wurde weiterhin deutlich, dass alle Unternehmen eine andere Vorstellung von der Netzwerkinitiative und entsprechenden Zielen hatten. Nicht die Gründung eines Netzwerkes mit vorhandener Idee, sondern die Initiierung eines Kommunikationsprozesses über die Rolle von Wasser für die Unternehmen und die Einbettung dieser Ressource im Rahmen einer nachhaltigen Entwicklung als Netzwerkziel wurde von allen Repräsentant/innen akzeptiert. Schließlich zeigte sich, dass eine Netzwerkidee, die sich auf die Erhaltung der Ressource als reines Wasser fokussiert, einen ersten Schritt in Richtung nachhaltige Entwicklung ermöglicht.
Das Schlussbild der Aufstellung zeigt, dass es verschiedene Ausrichtungen der großen Wasserwerke gibt, sodass vielfältige Aspekte miteinander zu klären sind. Das Element Nachhaltigkeit steht mit vielen Wasserversorgungsunternehmen und der Netzwerkidee in einer Klärungsposition, was bedeutet, dass ein Nachhaltigkeitsverständnis und eine klare Vision zu entwickeln sind. Die Angst vor Veränderung ist unter den großen Wasserunternehmen immer noch stark vorhanden, sodass Veränderungen auf organisatorischer Ebene anstehen. Es zeigt sich darüber hinaus, dass die Unternehmen eine strikte Kostenorientierung aufweisen und gegebene Strukturen und Infrastrukturen aufrechterhalten wollen. Keinesfalls wollen die Unternehmen mit verwässerten Nachhaltigkeitsfragen überfrachtet werden. Wasser als reine und natürliche Ressource sowie Nachhaltigkeit stehen in Bezug zueinander, die konkrete Ausprägung gilt es jedoch zu klären. Das Ethos der Wasserindustrie hat einen klaren Platz, wird jedoch nicht angeschaut und somit nicht direkt wahrgenommen (Abbildung 2).

Dieses Beispiel zeigt auf, dass die Anwendung einer SyStA und ein anschließender gemeinschaftlicher Reflexionsprozess, eine Neuausrichtung einer ursprünglichen Idee beziehungsweise Initiative sowie weitere Einblicke in die Widerstandsfähigkeit und das Ethos der Wasserwirtschaft ermöglichen. Die Wasserindustrie ist gut etabliert und auf Wachstum und Transformation fokussiert. Insgesamt werden Wissenszuwachs sowie konkrete Handlungsoptionen sichtbar. Nutzen und Erfolge von SyStA sind klar erkennbar: schnelle Problemerkennung und -strukturierung sowie neue Lösungen und mögliche Szenarien für Entscheidungen.

\section{Diskussion: Anwendung der Methode in Science-private-Partnerships}

Erkenntnisse, Umsetzungsoptionen und Schlussfolgerungen, die aus SyStA resultieren, sind durch ein reines Dokumentenstudium, Interviews oder eine empirische Erhebung häufig kaum erreichbar, auch nicht in einer vergleichbaren Schnelligkeit. Hinzu kommt das eigene Erleben eines Systemabbildes sowie der entsprechenden Wirkzusammenhänge. Kopp (2013) zeigt eine Kompetenzerhöhung des Denkens in multiplen Ursache-Wirkungs-Zusammenhängen. Die Methode zeigt sich so-

\begin{tabular}{lll}
\hline Einsatz in Lehre & Einsatz in Forschung & Einsatz in Praxis \\
\hline Gestaltung von Systemkompetenz & Suche nach Strukturen und Mustern & Strukturierung von Herausforderungen \\
\hline Visualisierung von Inhalten & Analyse von Beziehungen & Fokussierung von Fragestellungen \\
\hline Raumsprache als schnelle Orientierung & Aufzeigen von Spannungsfeldern & $\begin{array}{l}\text { Visualisierung von Systemkomponenten und } \\
\text {-gefüge }\end{array}$ \\
\hline Handlungslernen & Wissen über Tiefenstrukturen von Systemen & Explizieren verdeckten Wissens \\
\hline Verbindung von Wissenschaft und Praxis & Veränderung von Wissensmustern & Simulation von Interventionen und verschiedener \\
& & Lösungsoptionen \\
\hline Lernkontexte durch verschiedene Sinne motivieren & Hypothesenbildung & Reflexion funktionaler und dysfunktionaler \\
\hline Supervision & & Elemente \\
\hline
\end{tabular}

Tabelle 1: Einsatzmöglichkeiten von SyStA in Science-private-Partnerships 


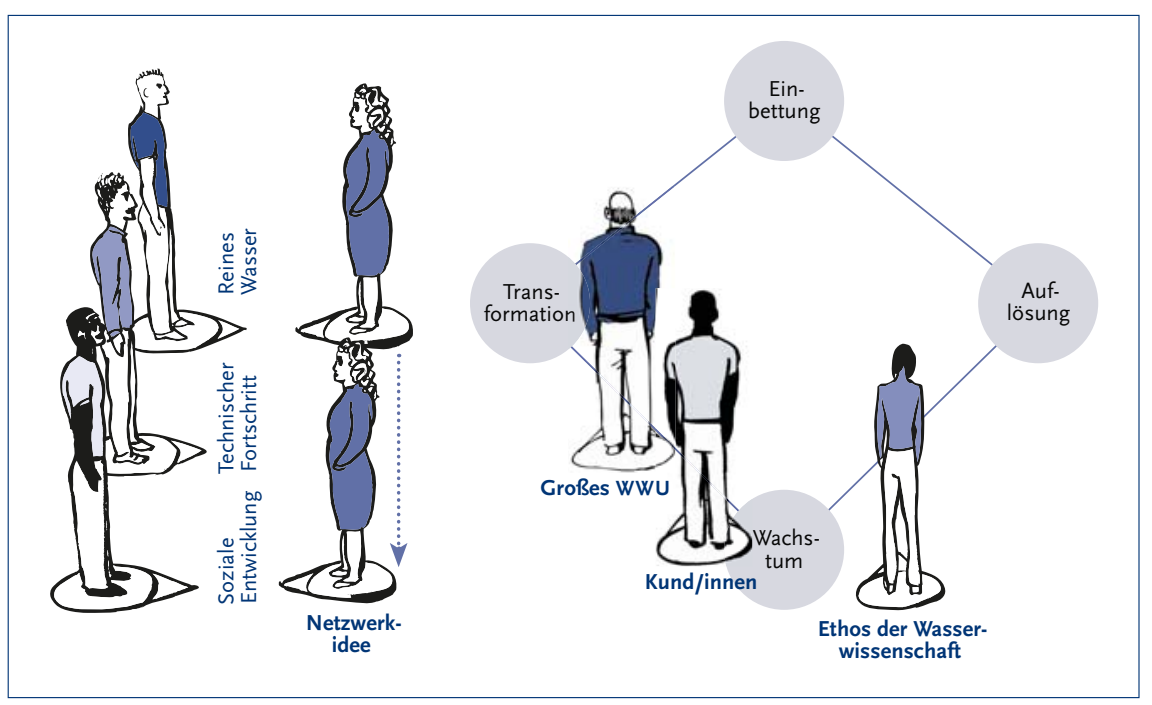

Abbildung 2: Links: Test der Netzwerkidee; Rechts: Polaufstellung ausgewählter Elemente der Wasserwirtschaft

mit als ein sehr wirkungsvolles Instrument bei der Erläuterung und der Vermittlung von Multikausalitäten in Systemen. Sie bietet sich als Ergänzung traditioneller Methoden zur Vermittlung von Systemkompetenz und zur Etablierung von Scienceprivate-Partnerships in sozialwissenschaftlichen sowie ökonomischen Kontexten an. Eine stärkere Lösungsausrichtung im Prozess könnte auch die Handlungsebene stärker integrieren. Zudem lässt sich zukünftig der Einsatz der Methode der Systemaufstellungen in der Hochschullehre zur Vermittlung systemischer Zusammenhänge mit anderen Lern- und Wissensvermittlungsmethoden kombinieren, umso Wahrnehmung und Verständnis sowie Transfer und Handlungen zugleich zu adressieren. Insbesondere dann, wenn Aufstellungen den geschlossenen organisationalen Raum verlassen und einen anschließenden Reflexionsprozess zwischen Praxisvertreter/innen, Forscher/innen und Studierenden ermöglichen, kann eine ganz neue Qualität nachhaltigkeitsbezogener Kompetenzen entstehen. Es gibt verschiedene Einsatzmöglichkeiten in Forschung, Lehre, Praxis (Kopp 2013), wie Tabelle 1 aufzeigt.
Allerdings führen Aufstellungen nicht zwingend $\mathrm{zu}$ systemischen und transdisziplinären Maßnahmen. Die Umsetzung der Erkenntnisse und des sich gezeigten neuen Wissens bedarf unternehmerischer Aktivitäten. Neben der Offenbarung verborgener oder nicht sichtbarer Muster ist für Science-private-Partnerships beziehungsweise den Prozess transdisziplinärer Forschung und Lehre die anschließende Diskussion von Unternehmensvertreter/innen, Forscher/innen und Studierenden über die sich gezeigten Beziehungsnetze und Interaktionsstrukturen von besonderer Bedeutung (Hisch 2008). Die Visualisierung der Tiefenstrukturen und verborgenen Muster ermöglicht eine Diskussion zwischen Wissenschaft und Praxis auf Augenhöhe sowie neue Ideen in Theorie und Praxis. Die Abbildung 2 zeigt eine mögliche Umsetzung einer Scienceprivate-Partnerships in Forschung und Lehre.

Praxis- und Unternehmensvertreter/innen sind positiv überrascht von den Möglichkeiten und der Substanz der Einblicke in und durch SyStA. Sie erleben die gemeinsame Reflexion bereichernd und nehmen viele Anregungen für ihre Tätigkeiten mit. Werden Studierende um Feedback zur Integration von Systemaufstellungen in Forschung und Lehre gebeten, sind die folgenden Antworten repräsentativ: „Es ist toll, dass wir die Chance haben, alternative Methoden auszuprobieren.“, „Die Methode ist eine großartige Ergänzung zu den traditionellen Methoden der Ökonomie.“ Allerdings sollte die Begeisterung der Studierenden und Praxis-Partner/innen für die Methode der Systemaufstellungen den verantwortungsvollen Umgang mit der Methode nicht ausblenden. Die Anwendung von SyStA in der transdisziplinären Forschung und Lehre setzt eine fundierte Ausbildung und einen respektvollen Umgang mit Menschen voraus. SyStA hat das Potenzial, Brücken zwischen innovativer Lehre, Forschung und Praxistransfer zu bauen hinsichtlich der Iden-

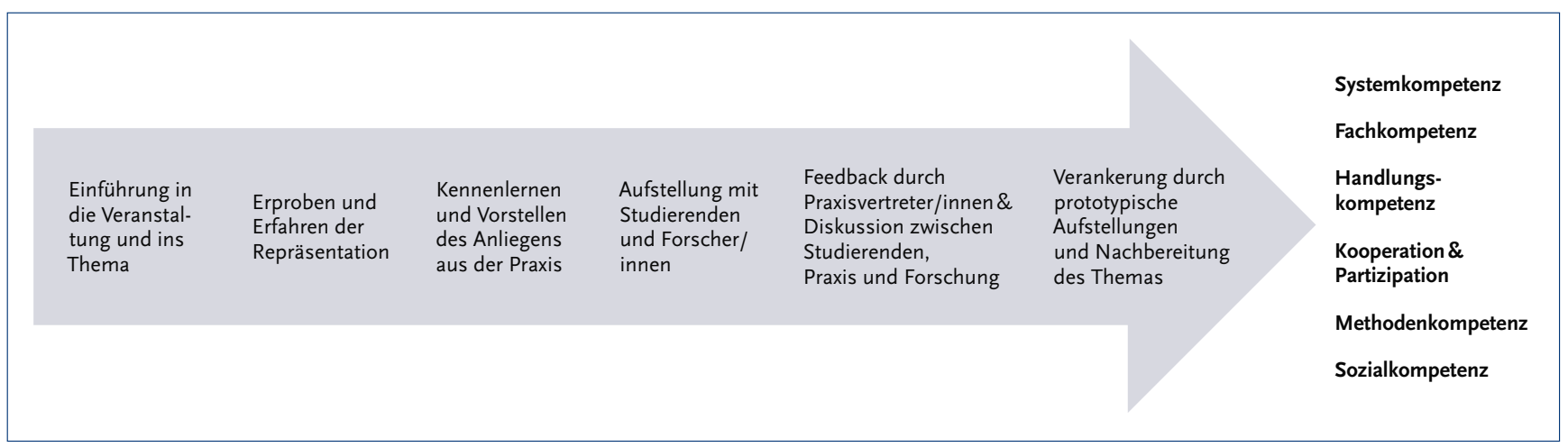

Abbildung 3: Verankerung von Science-private-Partnerships in Forschung und Lehre 
tifizierung und Initiierung neuer transdisziplinärer Optionen und Möglichkeiten für nachhaltigkeitsbezogene Maßnahmen und Umsetzungsszenarien. Gerade deshalb sind ein ethischer und respektvoller Umgang mit den Anliegen von Menschen und die Ausgestaltung der Prozesse zwingend. Erfahrungen aus den Science-private-Partnerships zeigen, dass eine Verknüpfung von Forschung und Lehre einerseits und organisationale Interessen andererseits in transdisziplinären Zusammenhängen einen essenziellen Beitrag zu einer nachhaltigen Entwicklung leisten können, gleichwohl die konkrete Realisierung der gewonnen Impulse in den Händen der Unternehmen oder Institutionen liegt.

\section{Fazit}

SyStA lassen sich in Forschung, Lehre und in Organisationen anwenden, um Herausforderungen, Konflikte oder Dilemmata im Kontext der Nachhaltigkeit lösungsorientiert zu fokussieren. Repräsentationen können für die Entdeckung neuer Perspektiven in allen Bereichen der Geistes- und Sozialwissenschaften genutzt werden. SyStA sind ein effektiver Weg, um komplexe Zusammenhänge, Herausforderungen auf mehreren Ebenen zu lehren und implizites Wissen zu konkretisieren und sichtbar zu machen. SyStA liefern wichtige Erkenntnisse, indem sie eine neue Dimension der menschlichen Wahrnehmung und eine neue Wissensqualität erfahrbar machen und im Raum abbilden. Die Integration der SyStA in Science-private-Partnerships ermöglicht es, komplexe Zusammenhänge, Herausforderungen auf mehreren Ebenen, nachhaltigkeitsbezogene transdisziplinäre Lehre und Forschung sowie die Bedeutung der Nachhaltigkeit für Unternehmen greifbar zu machen. Bei der Vermittlung von und Sensibilisierung für komplexe Themen wie Nachhaltigkeit lassen sich Lernfortschritte sowohl auf der Wissensebene erreichen als auch auf der Handlungsebene. Gleichwohl führen Systemaufstellungen nicht zwangsläufig zu systemischem Handeln; jedoch können sie ein Denken und Handeln in Mehrebenensystemen und multiplen Wirkungszusammenhängen stärken und schärfen. Eine Zusammenarbeit von Organisationen mit Forschungsinstitutionen geht über konventionelle Organisationsaufstellungen hinaus, da in Science-private-Partnerships mehr Formate für die Systemebene eingebracht werden können. Eine fundierte Reflexion auf Grundlage des Standes der Wissenschaft und entsprechender neuer Ansätze wird so erst möglich werden. Somit sind zugleich neue und schnelle Wege geebnet, innovative und nachhaltige Ideen aus der Forschung in die Praxis hinein zu transferieren und umzusetzen sowie neue Impulse aus der Praxis in die Nachhaltigkeitsforschung und -lehre zu integrieren.

\section{Literatur}

Daimler, R. (2014): Basics der Systemischen Strukturaufstellungen. München, Kösel-Verlag.

Franke, U. (2003): The River Never Looks Back. München, Carl-AuerSysteme Verlag.
Gehlert, T. (2014): GHZ - Theorem and Systemic Constellations. Quantum Teleportation in Multi-Particle Systems without Bell's Inequality. 10/2014: 1-6.

Greenberger, D. M. et al. (1990): Bell's Theorem without Inequalities. In: American Journal of Physics 58/12: 1131-1143.

Greenberger, D. M. et al. (2008): A Bell Theorem Without Inequalities for Two Particles. Using Efficient Detectors. In: Physical Review 78.

Hirsch, G. H. et al. (2008): Handbook of Transdisciplinary Research. Heidelberg, Springer.

König, O. (2007): Aufstellungsarbeit zwischen Supervision, Beratung, Therapie und Ideologie. In: Gruppendynamik und die Professionalisierung psychosozialer Berufe. Heidelberg, Carl-Auer Verlag. 150-176.

Kopp, U. (2013): Systemische Nachhaltigkeitskompetenzen für Führungskräfte. Erfahrungen mit Aufstellungsarbeit in der Managementausund -weiterbildung. In: Die Unternehmung 67/2: 127-154.

Lamnek, S. (1988): Qualitative Sozialforschung. Band 1: Methodologie. München, Psychologie Verlags Union.

Mayring, P. (2002): Qualitative Sozialforschung. 5. Auflage. Weinheim, Beltz Verlag.

Müller-Christ, G. (2013): Wo stehen Nachhaltigkeit und Ressourcenorientierung im Unternehmen? Ordnungsangebote im Prämissengerangel durch Systemaufstellungen. In: Klinke, S./Rohn, H. (Hrsg.): RessourcenKultur: Vertrauenskulturen und Innovationen für Ressourceneffizienz im Spannungsfeld normativer Orientierung und betrieblicher Praxis. Baden Baden, Nomos. 383-392.

Reichertz, J. (1999): Gültige Entdeckung des Neuen?: zur Bedeutung der Abduktion in der qualitativen Sozialforschung. In: Österreichische Zeitschrift für Soziologie. 48/4: 47-64.

Rosenberg, M. B. (2013): Gewaltfreie Kommunikation. Eine Sprache des Lebens. Gestalten Sie Ihr Leben, Ihre Beziehungen und Ihre Welt in Übereinstimmung mit Ihren Werten. 11. Auflage. Paderborn, Junfermann Verlag.

Schlötter, P. (2005): Vertraute Sprache und ihre Entdeckung. Systemaufstellungen sind kein Zufallsprodukt - der empirische Nachweis. Heidelberg, Carl-Auer Verlag.

Sheldrake, R. (1988): The Presence of the Past. London, Blond \& Briggs.

Sheldrake, R. (2001): Das Morphologische Feld sozialer Systeme. In: Weber, G. (Hrsg.): Derselbe Wind lässt viele Drachen steigen. Systemische Lösungen im Einklang. Heidelberg, Cal-Auer Verlag. 29-42.

Sparrer, I./Varga von Kibéd, M. (2010): Klare Sicht im Blindflug. Schriften zur Systemischen Strukturaufstellung. Heidelberg, Carl-Auer Verlag.

Sparrer, I./Varga von Kibéd, M. (2001): Systemische Strukturaufstellungen. Simulation von Systemen. In: Lernende Organisation 4. 6-14.

Varga von Kibéd M./Sparrer I. (2014): Ganz im Gegenteil. Tetralemmaarbeit und andere Grundformen Systemischer Strukturaufstellungen für Querdenker und solche, die es werden wollen. 8. Auflage. Heidelberg, Carl-Auer Verlag.

Wade, H. (2004): Systemic working: the constellations approach. In: Industrial and Commercial Training 36/5: 194-199.

Walker B. et al. (2002): Resilience Management in Social-ecological Systems: a Working Hypothesis for a Participatory Approach. In: Conservation Ecology 6/1. Art. 14.

\section{AUTORIN + KONTAKT}

PD Dr. Marlen Arnold ist Projektleiterin von mint.online an der Universität Oldenburg und Inhaberin von Naturelnspires. Ihre Forschungsschwerpunkte sind Nachhaltigkeitsmanagement und Nachhaltigkeitsinnovationen.

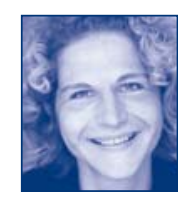

Carl von Ossietzky Universität Oldenburg, Arbeitsbereich Weiterbildung \& Bildungsmanagement, 26111 Oldenburg. E-Mail: marlen.arnold@uni-oldenburg.de 